Article - Human and Animal Health

\title{
Pre-treatment by Rosemary Extract or Cell Transplantation Improves Memory Deficits of Parkinson's Disease: When Tradition Meets the Future
}

\section{Seyyede Narjes Hashemi Rad ${ }^{1}$}

https://orcid.org/0000-0002-2889-9893

\section{Maryam Haji Ghasem Kashani ${ }^{1 *}$}

https://orcid.org/0000-0001-8889-6427

Kataneh Abrari ${ }^{1}$

https://orcid.org/0000-0002-8056-9218

1Damghan University, Department of Cellular and Molecular Biology, School of Biology and Institute of Biological Sciences, Damghan, Iran.

Editor-in-Chief: Paulo Vitor Farago

Associate Editor: Paulo Vitor Farago

Received: 2018.07.27; Accepted: 2020.10.07.

*Correspondence: kashani@du.ac.ir; Tel: +98-23-35220242; (M.H.G.K.).

\section{HIGHLIGHTS}

- Spatial learning and memory impairment through bilateral injection of 6-OHDA (6Hydroxydopamine) into the SNc which also leads to the destruction of neurons in the hippocampus.

- ADSCs (Adipose tissue-derived stem cells) transplantation had neuroprotective effect and recovered the memory deficit in 6-OHDA-induced rat model of Parkinson's disease.

- Rosemary extract pre-treatment compensated the memory deficit in 6OHDA-induced rat model of Parkinson's disease.

ABSTRACT: The therapeutic effect of adipose tissue-derived stem cells (ADSCs) or RE on hippocampal neurogenesis and memory in Parkinsonian rats were investigated. Male rats were lesioned by bilateral intra-nigral injections of 6-OHDA and divided into six groups: 1. Lesion 2 and 3: RE and water groups were lesioned rats pretreated with RE or water, from 2weeks before neurotoxin injection and treated once a day for 8 weeks post lesion. 4\&5: Cell and $\alpha$-MEM ( $\alpha$-minimal essential médium) received intravenous injection of BrdU-labeled ADSCs or medium, respectively from 10days post lesion until 8weeks later. 6: Sham was injected by saline instead of neurotoxin. Memory was assessed using Morris water Maze (MWM), one week before and at 1, 4 and 8weeks post 6-OHDA lesion. After the last probe, the animals were sacrificed and brain tissue obtained. Paraffin sections were stained using cresyl violet, anti-BrdU (Bromodeoxyuridine / 5bromo-2'-deoxyuridine), anti-GFAP (Glial fibrillary acidic protein) and anti-TH antibodies- There was a 
significant difference of time spent in the target quadrant between groups during probe trial at 4 and 8 weeks' post- lesion. Cell and RE groups spent a significantly longer period in the target quadrant and had lower latency as compared with lesion. Treated groups have a significantly higher neuronal density in hippocampus compared to water, a-MEM and lesion groups. BrdU positive cells were presented in lesioned sites. The GFAP (Glial fibrillary acidic protein) positive cells were reduced in treated and sham groups compared to the water, $\alpha-M E M$ and lesion groups. Oral administration of RE (Rosemary extract) or ADSCs injection could improve memory deficit in the Parkinsonian rat by neuroprotection.

Keywords: Rosemary extract; cell transplantation; hippocampus; memory.

\section{INTRODUCTION}

Parkinson's disease (PD) is a progressive neurodegenerative disease and occurs in $1 \%$ of people over the age of 55. Patients suffer from motor impairment which is caused by severe lack of dopamine in the SNc and functional disruption of the basal nuclei. An important neurological feature is the degeneration of dopaminergic neurons in the substantia nigra pars compacta (SNc) followed by the deprivation of dopamine in the striatum [1]. Recent studies have shown that $48 \%$ of patients with PD had memory loss, one of the first symptoms, and $48 \%$ of them had a dementia after 15 years [2]. There is no definite evidence about the probability of the disease. Probably, aging and genetics as well as oxidative stress, protein accumulation, mitochondrial dysfunction, external toxicity, inflammatory immune responses and environmental factors such as physical trauma and infection are involved in the onset of the disease and deterioration of SNc [3]. Loss of dopamine in the hippocampus affects spatial learning and memory. The effect of dopamine on memory is thought to play an important role in synaptic plasticity and memory mechanisms of the hippocampus [4]. In addition to pharmacological treatments, medicinal plants and cell therapy are the most important ways to treat PD symptoms. The long term use of L-Dopa (a precursor of dopamine) has been associated with dyskinesia and reduction of efficacy, which requires an increase in the amount of drug to reduce the complications of the disease. Rosemarinus officinalis leaves extract (RE) is one of the medicinal herbs with therapeutic potential to control the PD symptoms [5]. RE contains phenolic compounds that have antioxidant, anti-allergic, anti-inflammatory and anti-apoptotic effects [6]. It has protective effects on the 6-OHDA and MPTP-induced oxidative stress and apoptosis in vitro, and neuroprotective effect on dopaminergic neurons in PD [7]. Ito and colleagues also reported in 2008 that rosemarinic acid (one of the compounds of the RE), like an antidepressant, stimulates the proliferation of neurons in the dentate gyrus of the hippocampus. They also revealed the anti-apoptotic effect of rosemarinic acid on astrocytes [8]. It has been observed that carnosic acid regulate autophagy and attenuate 6-OHDA-induced neurotoxicity in vitro [9]. Transplantation of dopaminergic cells into striatum is a laboratory method that have overcame the drug treatments and is used in the improvement of PD syndrome by providing an unlimited source of dopamine synthesis through the transplanted cells [10]. The use of embryonic stem cells has many limitations, while adult stem cells override these constraints. Adult stem cells are undifferentiated cells of the body and replaced lost cells to treat neurodegenerative diseases [11]. Mesenchymal stem cells (MSCs) are a type of adult stem cells. These cells are easily accessible and can be isolated from adipose tissue and bone marrow [12]. Adipose tissue-derived stem cells (ADSCs) have the ability to differentiate into different cell types of the body. These cells survive and migrate to the lesion site and differentiate into neurons after transplantation [13]. It was also shown that ADSCs can repair the damaged tissues by secreting cytokines and growth factors through paracrine pathways [14]. Transplanted ADSCs have the ability to secrete glial-derived neurotropic factor (GDNF) and increase the neuronal viability of the hippocampus [15].

In this study, we compared the therapeutic efficacy of RE and ADSC on the memory impairment of PD. Since the hippocampus receives dopaminergic input from the SNc [16], the destruction of dopaminergic neurons and subsequent dopamine depletion of the hippocampus can lead to memory deficits [17]. Released dopamine from SNc affects the neurogenesis in the hippocampal dentate gyrus and regulates memory formation, due to the relationship between neurogenesis and memory formation [18]. In this study, bilateral injection of 6-OHDA into the SNc of male rats caused memory impairment similar to that seen in PD. Then, the neuroprotective effect of intravenous transplantation of ADSCs or oral administration of RE on the hippocampus and memory improvements were investigated. 


\section{MATERIALS AND METHODS}

\section{Animals}

Male wistar rats weighing 220-280 g (n: 48) were purchased from Razi Research Institute, Karaj, Iran and housed at $20-24{ }^{\circ} \mathrm{C}$ under a 12 hour light/dark cycle, with free access to food and water. All experiments were done in accordance with the National Institute of Health Guide for the care and use of laboratory animals (NIH Publication No. 23-80, revised 1996) and approved by the research ethical standards in Damghan University.

\section{Experimental groups}

Animals were single-housed and kept separate for one week. Then they were trained in MWM apparatus, as we explained it in bellow. At the end of the second week, animals were positioned in a stereotaxic frame and 6-OHDA (Sigma-Aldrich, USA, CAS NO. 636-00-0) or normal saline was injected bilaterally into the SNc at specific coordinates, depended of the group. One week after injection, the second MWM training took placed. Ten days after injection of 6-OHDA, lesioned rats in cell group (Figure 1) were transplantated intravenously by BrdU- labeled ADSCs or a-MEM. The animals were subjected to two additional MWM tests, five and nine weeks after injection of 6-OHDA or normal saline. Decapitation, histological and immunohistochemical studies were done at the final week of experiments. Animals were gavaged by water or RE from 2 weeks before, until 8 weeks after the injection of 6-OHDA or normal saline. The experimental design were showed in detail in Figure 1.

Then six experimental groups were named as follows:

Lesion: 6-OHDA (6 $\mu \mathrm{g} / 2 \mu \mathrm{L}$ of normal saline) was injected bilaterally into the SNc.

Sham: animals which received $2 \mu \mathrm{L}$ of normal saline instead of neurotoxin.

$\mathrm{RE}$ : lesioned rats were pretreated daily with $\mathrm{RE}(50 \mathrm{mg} / \mathrm{kg})$, from two weeks prior to neurotoxin injection up to eight weeks post-injection.

Cell: lesioned rats transplanted intravenously by BrdU (Bromodeoxyuridine / 5-bromo-2'-deoxyuridine) labeled ADSCs (106 cells per $500 \mu \mathrm{L}$ of medium), from 10 days post lesion until 8 weeks later.

Water: lesioned rats were gavaged by water as RE vehicle from 14 days before until 8 weeks after the lesion.

a-MEM: lesioned rats were administered intravenously by $500 \mu \mathrm{L}$ of medium.

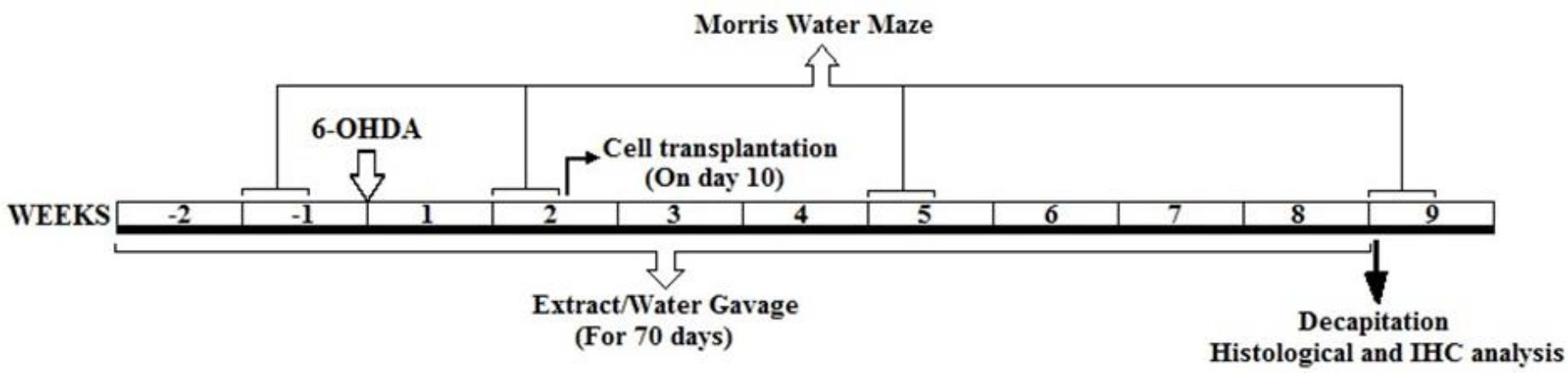

Figure 1. Experimental design. Timeline showing the behavioral and histological studies along with treatments.

\section{Preparation of memory impairment model using 6-OHDA}

Rats were anesthetized with ketamine $(100 \mathrm{mg} / \mathrm{kg})$ and xylazine $(10 \mathrm{mg} / \mathrm{kg})$, and placed on a stereotaxic frame (Stoelting, 1886, USA). A total of six $\mu$ g 6-OHDA hydrobromide was dissolved in $2 \mu \mathrm{L}$ of sterile saline containing $0.2 \%$ ascorbic acid and injected bilaterally into the SNc with a Hamilton syringe (26 gauge, Hamilton, Massy, France) at a flow rate of $1 \mu \mathrm{L} / \mathrm{min}$. Stereotaxic coordinates from the bregma were: (anteroposterior $-0.5 \mathrm{~mm}$, mediolateral $\pm 2.1 \mathrm{~mm}$, and dorsoventral $-7.7 \mathrm{~mm}$ ) [19]. The syringe was left in place for 5 min after injection and then removed slowly to optimize toxin diffusion.

\section{Morris Water Maze}

The Morris water maze is an open circular pool $(190 \mathrm{~cm}$ in diameter and $60 \mathrm{~cm}$ in height) that is filled approximately half-way with water $\left(24 \pm 1^{\circ} \mathrm{C}\right)$. A circular platform was hidden in the center of the southeast quadrant of the maze, three centimeters below the water level. In order to navigate, spatial clues in four 
directions of the maze were installed on the walls of the room. Each animal was randomly placed into the water in one of four directions of north, south, east or west to find the hidden escape platform. If the animal did not find the platform within a minute, they were guided to the platform and left on the platform for 20 seconds. Training sessions continued for four days, each day four trials, and the animal's performance was recorded using a video camera connected to a computer. The memory was evaluated 24 hours after the completion of the training with a probe trial of 60 seconds. At this stage, after removing the platform, the animal was placed into the water from the opposite quadrant of the platform. Escape latency, distance moved, average speed and the time spent in the target quadrant were measured and analyzed using EthoVision XT software.

\section{Rat ADSCs isolation and culture}

The interscapular fat tissues were collected from rats (6-8 weeks age), under sterile conditions and placed in a sterilized culture dish under laminar hood. Fat tissues were digested mechanically and enzymatically with $0.075 \%$ collagenase type-I (Sigma-Aldrich; CAS NO.9001-12-1) for 1 hour at $37^{\circ} \mathrm{C}$. ADSCs were isolated, based on their capacity to adhere to the plastic flask. Isolated cells were incubated with $10 \%$ fetal bovine serum (FBS, Gibco, cat.No. 12203C) containing a-MEM (a-minimal essential medium; Gibco/BRL; cat.No. 52100-0.3), and 1\% penicillin/streptomycin (Gibco; cat.No. 15140). First passage of cells was performed at $80-90 \%$ confluency. P4 cells were prepared for transplantation.

\section{BrdU labeling and cell transplantation}

The cells were incubated in medium containing $10 \mu \mathrm{M}$ bromodeoxyuridine (BrdU, Sigma; CAS NO.5914-3 ) for $48 \mathrm{~h}$ and approximately labeled cells $\left(1 \times 10^{6} / 500 \mu \mathrm{l}\right.$ medium) transplanted by injection into the tail vein, at 10 days after lesion.

\section{Rosemary extract preparation}

$\mathrm{RE}$ containing $40 \%$ of carnosic acid (carnosic acid powder CAP25-110401) was purchased from "Hunan Geneham Biomedical Technology, China" and dissolved in distilled water.

Based on previous studies, effective dose of RE was $50 \mathrm{mg} / \mathrm{kg}$ [20-22]. Rats were gavaged orally with extract at a dose of $50 \mathrm{mg} / \mathrm{kg}$.

\section{Tissue preparation and immunohistochemistry}

After 8 weeks, the animals were deeply anesthetized and transcardial pefusion of phosphate buffered saline (PBS) and 4\% paraformaldehyde solution were carried out (100 ml of PBS followed by $200 \mathrm{ml}$ of $4 \%$ Paraformaldehyde into the left ventricle). The brains were quickly extracted from the skull and fixed with $4 \%$ paraformaldehyde for 24 hours. coronal microscopic sections of 10 micron thickness were prepared at the level of $+2.8 \mathrm{~mm}$ relative to the bregma (within the areas of DG, CA1 and CA3 of hippocampus) and collected onto glass slides. The sections were stained with $0.75 \%$ Cresyl violet and cell counting was performed with X400 magnification. The number of neurons in the regions of DG, CA1 and CA3 were counted in five fields with an area of $400 \mu \mathrm{m}^{2}$ and the means were calculated; the cell counting was repeated 7 times. Then the slides were photographed with $\times 400$ magnification using a digital camera (Nikon, DXM 1200, USA) equipped a fluorescent microscope (E600-Eclipse Nikon, Japan). In order to confirm the destruction of the dopaminergic neurons of the SNc, an anti-Tyrosine hydroxylase (TH) staining was performed. Some sections of SNc and hippocampus regions were boiled in citrate buffer in order to antigen retrieval and incubated with the primary antibodies: anti-TH (Anti-Tyrosine Hydroxylase, MilliporeSigma-AB152); anti-BrdU (Monoclonal anti-BrdU antibody in mouse, Sigma-Aldrich, B2531); antiGFAP (Anti-Glial Fibrillary Acidic Protein, Sigma-Aldrich, G3893) overnight at $4{ }^{\circ} \mathrm{C}$. Samples were incubated with the secondary anti-bodies conjugated with HRP for TH and BrdU (Abcam, ab205718) for 1 hour at $37^{\circ} \mathrm{C}$, followed by incubation with $0.1 \%$ DAB (Sigma-Aldrich, D7304) substrate solution for 15 minute. For anti-GFAP immunostaining, sections were incubated for 1 hour at $4^{\circ} \mathrm{C}$ with fluorescein conjugated secondary antibody (Abcam, ab97029). The DAB $0.1 \%$ substrate solution was used and sections were dehydrated and mounted using DPX.

\section{Statistical analysis}


Data were analyzed by SPSS 23 software, using one-way ANOVA or Paired-sample t-test. Tukey's post hoc test was used for analyzing the data within groups. The results are indicated as mean \pm standard error of the mean (SEM) and considered as meaningful at $p \leq 0.05$.

\section{RESULTS}

\section{Behavioral analyses}

Animals of all the groups underwent a 4-day learning period one week before and one, four and eight weeks after the lesion. Paired sample t-test showed a significant difference in escape latency and distance traveled between the first and the fourth day in all the groups $(p<0.05)$, meaning that all animals were able to learn the task. One-way ANOVA showed no significant difference among the groups in escape latency, one week before the lesion, for four training days, $\left(F_{(5,42)}=1.12, P=0.36\right),\left(F_{(5,42)}=0.36, P=0.873\right)$, $\left(F_{(5,42)}=9.52, P=0.733\right)$ and $\left(F_{5,42)}=1.45, P=0.213\right)$ respectively (Figure 2.a). Also there were no significant difference among the groups in distance traveled for four training days, $\left(F_{(5,42)}=1.96, P=0.105\right)$, $\left(F_{(5,42)}=3.28, P=0.114\right),\left(F_{(5,42)}=1.17, P=0.336\right)$ and $\left(F_{5,42)}=1.05, P=0.398\right)$ respectively. In the second learning period (one week after the lesion), a significant difference in escape latency, was observed using one-way ANOVA in days 1, 2, 3 and 4; $\left(F_{(5,42)}=110.31, P=0.000\right),\left(F_{(5,42)}=187.57, P=0.000\right),\left(F_{(5,42)}=\right.$ 93.8, $\mathrm{P}=0.000)$ and $\left(\mathrm{F}_{5,42)}=143.09, \mathrm{P}=0.000\right)$ respectively, and in distance traveled for four training days, $\left(F_{(5,42)}=61.91, P=0.000\right),\left(F_{(5,42)}=159.5, P=0.000\right),\left(F_{(5,42)}=47.98, P=0.000\right)$ and $\left(F_{5,42)}=52.28, P=\right.$ 0.000 ) respectively. Tukey's post hoc test showed that animals in the lesion, water, cell and a-MEM groups had significantly higher escape latency and distance traveled in comparison with the sham and extract groups (Figure 2.b).

In the third learning periods (4 weeks after the lesion), one-way ANOVA showed a significant difference in days one through four, in escape latency $\left(F_{(5,42)}=33.05, P=0.000\right),\left(F_{(5,42)}=92.84, P=0.000\right),\left(F_{(5,42)}=\right.$ 121.33, $P=0.000)$ and $\left(F_{5,42)}=60.0, P=0.000\right)$ respectively, and in distance traveled $\left(F_{(5,42)}=48.67, P=\right.$ $0.000),\left(F_{(5,42)}=65.74, P=0.000\right),\left(F_{(5,42)}=122.54, P=0.000\right)$ and $\left(F_{5,42)}=47.66, P=0.000\right)$ respectively. Results of the fourth learning periods ( 8 weeks after the lesion) were as same as third learning trials; results of escape latency respectively from first to fourth trial days were $\left(F_{(5,42)}=334.47, P=0.000\right),\left(F_{(5,42)}=\right.$ 396.52, $P=0.000),\left(F_{(5,42)}=300.8, P=0.000\right)$ and $\left(F_{5,42)}=569.97, P=0.000\right)$, and results of distance traveled were $\left(F_{(5,42)}=188.53, P=0.000\right),\left(F_{(5,42)}=130.08, P=0.000\right),\left(F_{(5,42)}=180.48, P=0.000\right)$ and $\left(F_{5,42)}=261.41, P=0.000\right)$.

Tukey's post hoc tests for third and fourth learning periods showed that sham, extract and cell groups performed significantly better $(p<0.000)$ than the water, lesion and a-MEM groups in escape latency as well as distance traveled (Figure 2.c and 2.d). At the end of third and fourth learning periods, a probe trial was taken to assess the spatial memory formation. During the 60-second probe trial, total distance traveled and time spent in the target quadrant and platform location latency were recorded. One-way ANOVA on time spent in the target quadrant, showed a significant difference among groups, both 4 and 8 weeks after the lesion $\left(F_{(5,42)}=12.44, P=0.000\right)$ and $\left(F_{5,42)}=86.33, P=0.000\right)$ (Figure 2.e). ANOVA on total distance traveled, showed no significant difference during the probe trials among the groups, 4 and 8 weeks after the lesion $\left(F_{(5,42)}=1.16, P=0.343\right)$ and $\left(F_{5,42}=1.25, P=0.3\right)$. One-way ANOVA on platform location latency data indicated a significant difference among the groups $\left(F_{(5,42)}=107.97, P=0.000\right)$ and $\left(F_{5,42)}=143.48, P=\right.$ 0.000 ) 4 and 8 weeks after the lesion respectively (data's not shown). Analysis followed by Tukey's post hoc test showed that cell and extract groups spent a significantly longer period in the target quadrant and had lower platform location latency as compared with lesion group, both at 4 and 8 weeks after the lesion $(p<0.05)$ (Figure 2.e). 
a) Escape latency and distance traveled, one week before the lesion
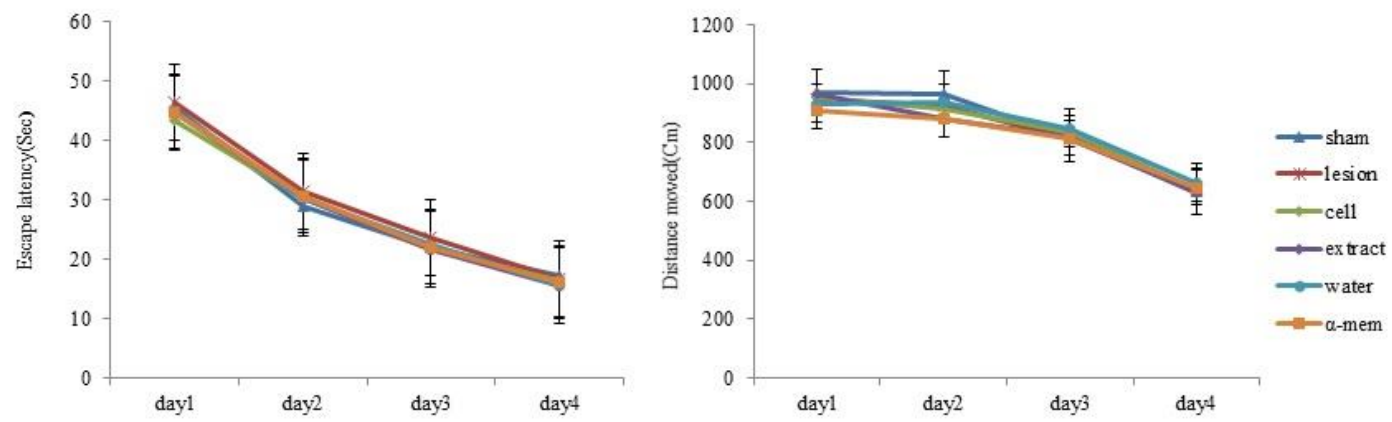

b) Escape latency and distance traveled, one week after the lesion
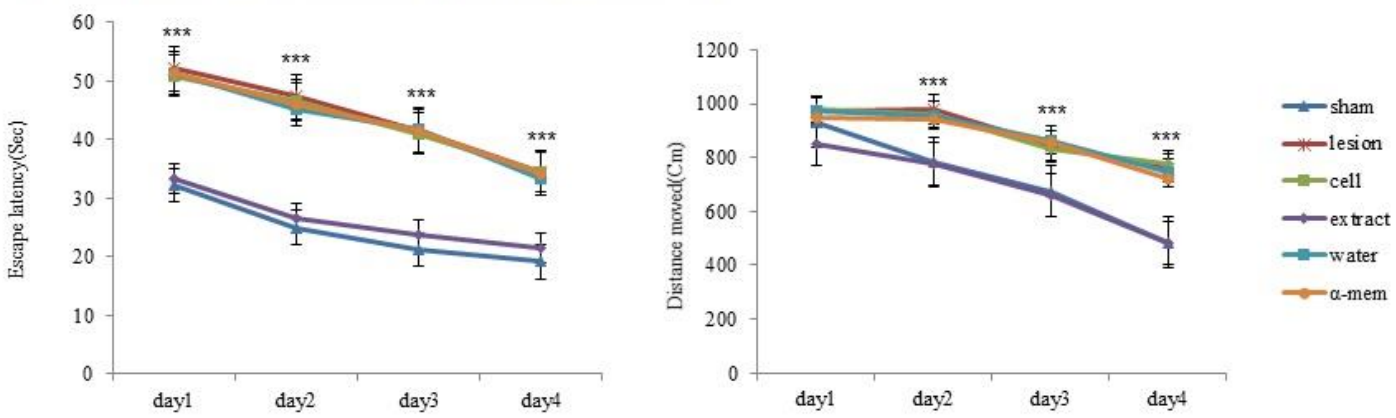

c) Escape latency and distance traveled, 4 weeks after the lesion
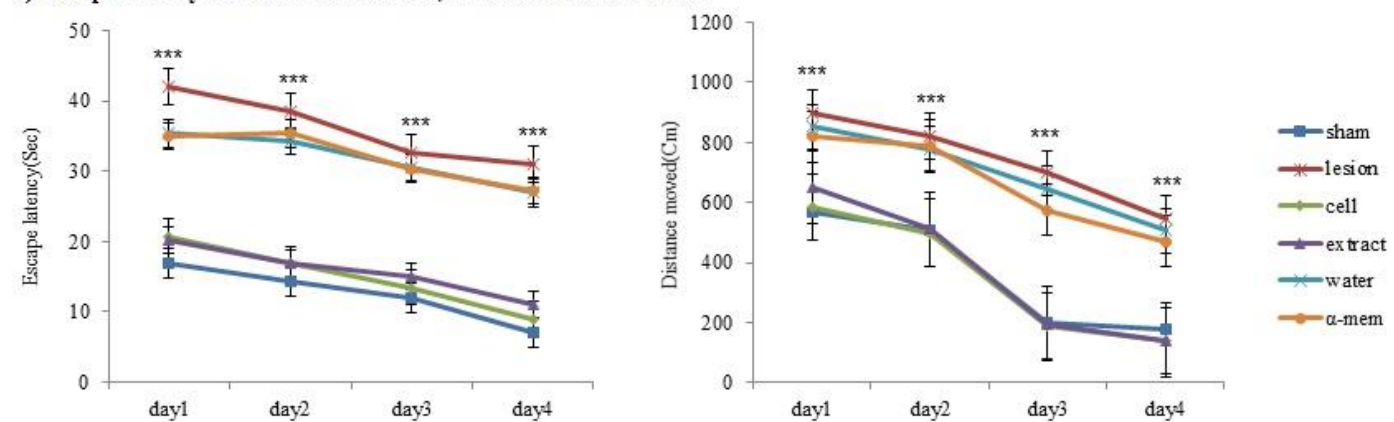

d) Escape latency and distance traveled, 8 weeks after the lesion 1000
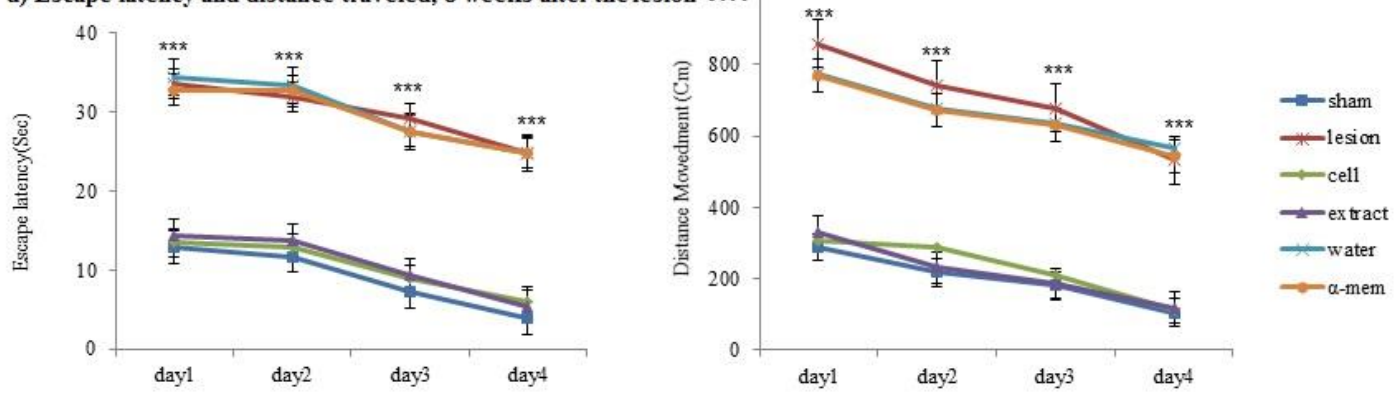

e) $\%$ Time spent in the target quadrant during the probe trials, 4 and 8 weeks after the lesion
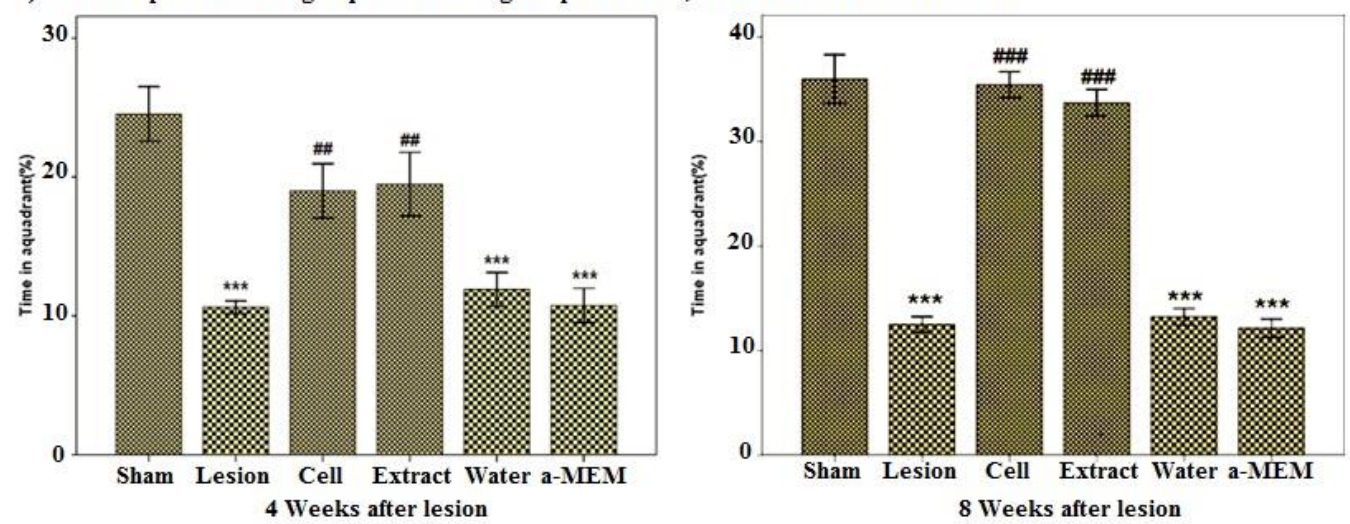

Figure 2. Morris water maze spatial learning and memory task. Escape latency and distance moved to reach the hidden platform in different training phases (a-d). Time spent in the target quadrant during the probe trials, 4 and 8 
weeks after the lesion (e). ${ }^{* * *} p<0.000$ compared with sham. \#\#\# $p<0.000, \# \# p<0.00$ compared with lesion. Data are presented as mean \pm SEM.

\section{Cresyl violet staining and Immunohistochemistry}

Immunohistochemistry was used to determine TH-positive neurons in the SNc of all groups. There were reduction of TH-positive neurons in the lesion, Water and a-MEM groups compared to Sham, Cell and Extract groups, but it was decreased in the SNc of lesion group (Figures 3, A-F).

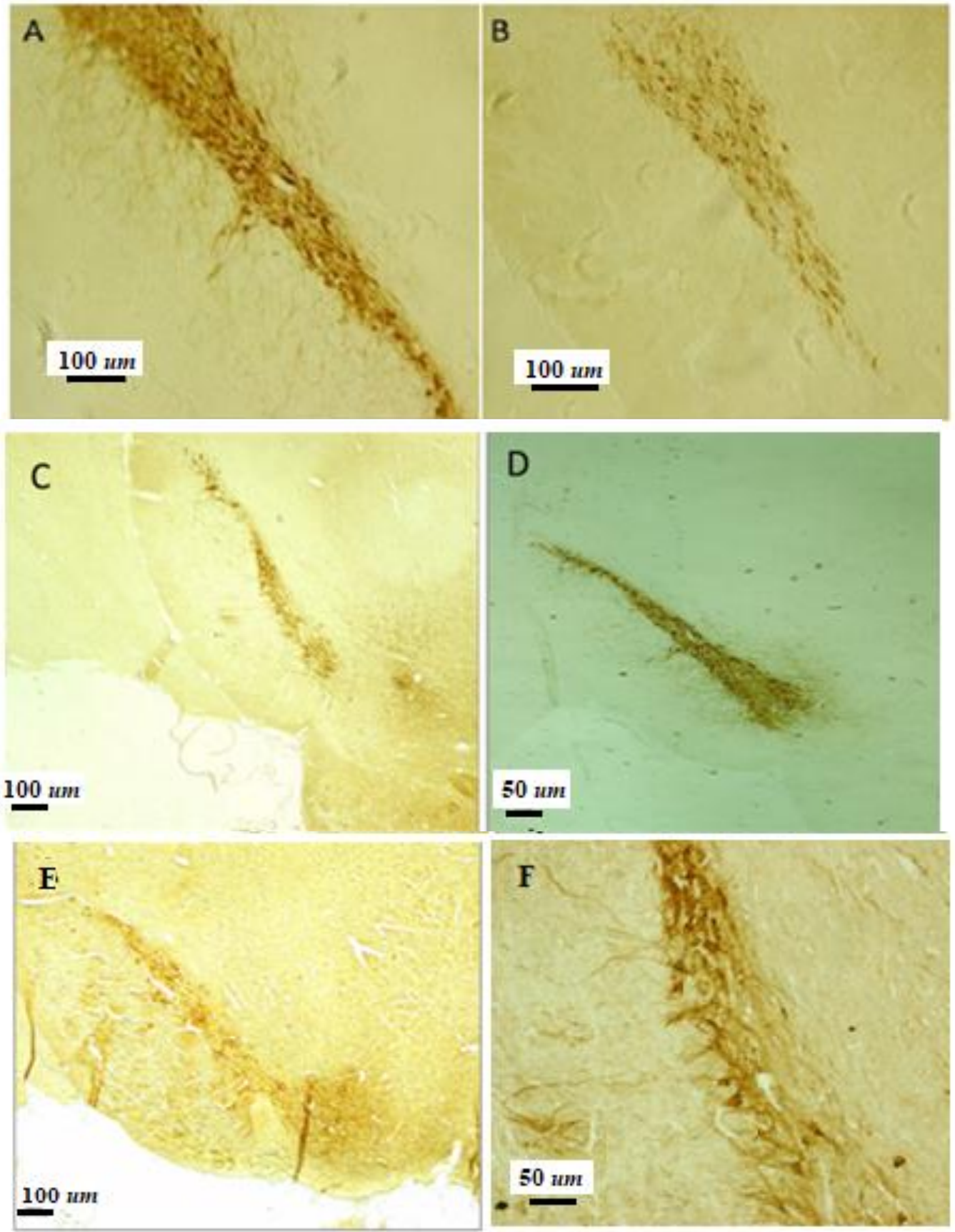

Figure 3. Immunohistochemical staining of TH-positive neurons in the SNc of Sham (A), Lesion (B), a-MEM (C), Cell (D), Water (E) and Extract (F) groups.

Cresyl violet staining showed the effect of neurotoxin 6-OHDA on neurons density in DG, CA1 and CA3 regions of the hippocampus, and also the protective effect of $R E(50 \mathrm{mg} / \mathrm{kg})$ and ADSCs transplantation (Figure 4). 


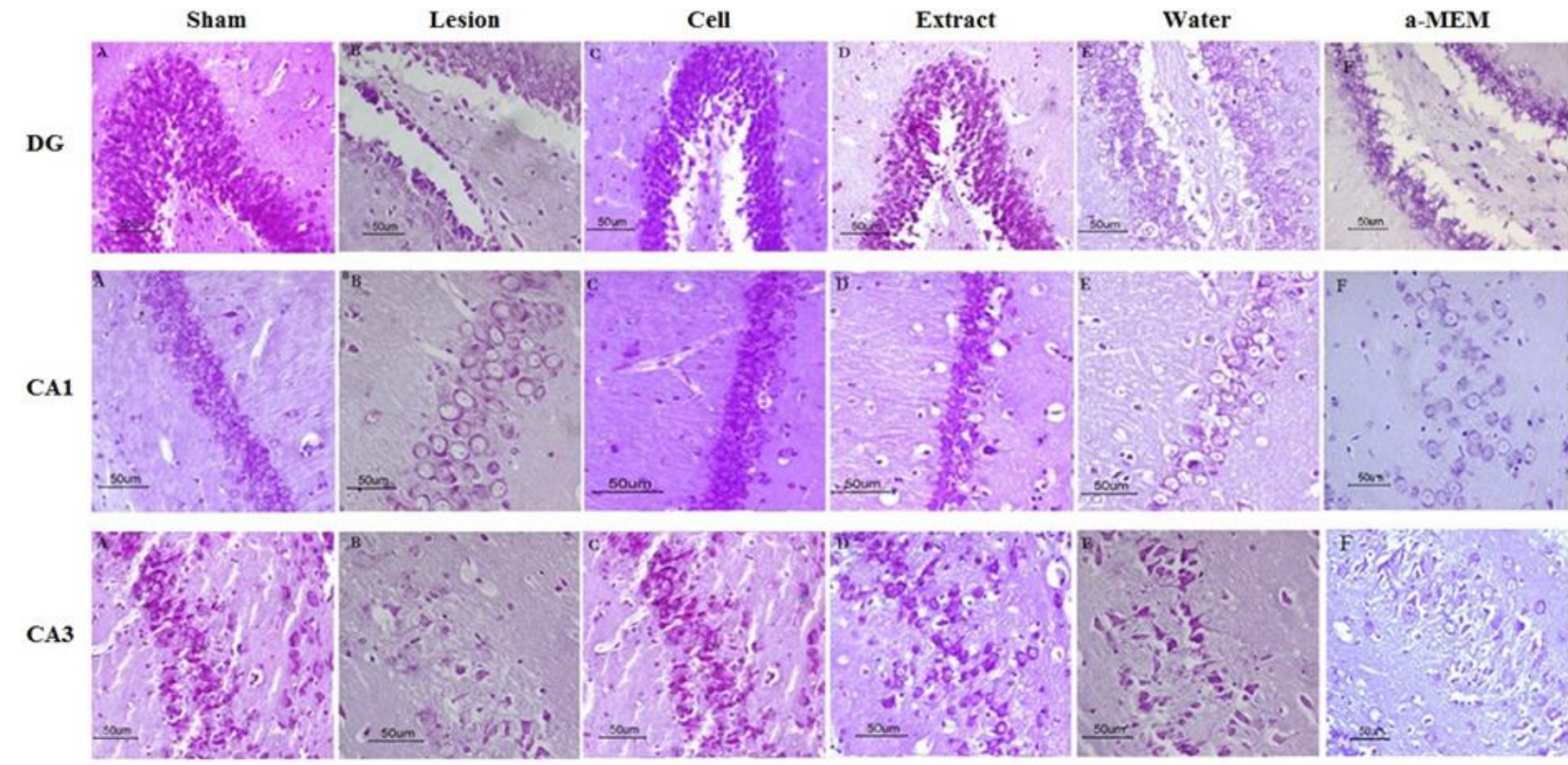

Figure 4. Cresyl violet staining of DG, $C A 1$ and $C A 3$ regions of the hippocampus in all groups. The neurns density of cell and extract groups were more than the other groups except the sham.

The density of neurons in the three regions of DG, CA1 and CA3 were quantitatively studied. The number of DG granular cells were significantly increased $(p<0.000)$ in the cell and extract groups, compared to lesion, water and a-MEM groups (Figure 5.a). Moreover, extract treatment and cell transplantation significantly increased $(\mathrm{p}<0.000)$ the number of CA1 and CA3 pyramidal neurons (Figure 5. $b, c)$.
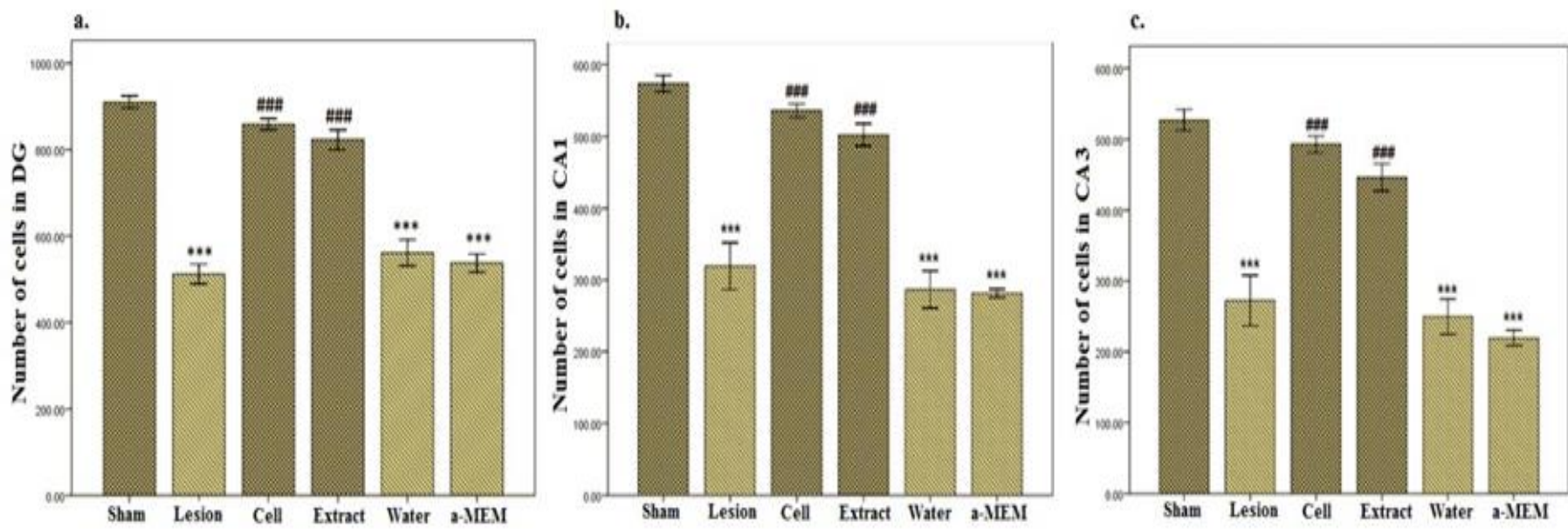

Figure 5. Cell count of Cresyl violet stained sections in DG (a), CA1 (b) and CA3 (c) of the hippocampus. The number of DG granular cells were significantly increased in the cell and extract groups, compared to lesion, water and a-MEM groups (a). Extract treatment and cell transplantation also significantly increased the number of CA1 and CA3 pyramidal neurons $(b, c) .{ }^{* * *} p<0.000$ compared with sham. \#\#\# $p<0.000$ compared with lesion. Data are presented as mean \pm SEM.

CD71 and CD90 ( MSCs specific positive markers ) positive ADSCs were shown in (Figure 6.a, b). BrdU-positive cells were also checked by anti-BrdU immunostaining, the labeled ADSCs successfully passed through blood brain barrier and successfully migrated to lesioned site (Figure 6.c).

As astrocytes activity and astrogliosis, are considered one of the main characteristics of PD, we investigated the density of astrocytes using anti-GFAP immunohistochemistry (Figures 7, a-f). GFAP positive cells in the DG (Figure 8.a) and SGZ (Figure 8.b) regions showed that the density of astrocytes in lesion, water and $\alpha-M E M$ increased significantly $(p<0.000)$ compared to sham, cell and extract groups. 

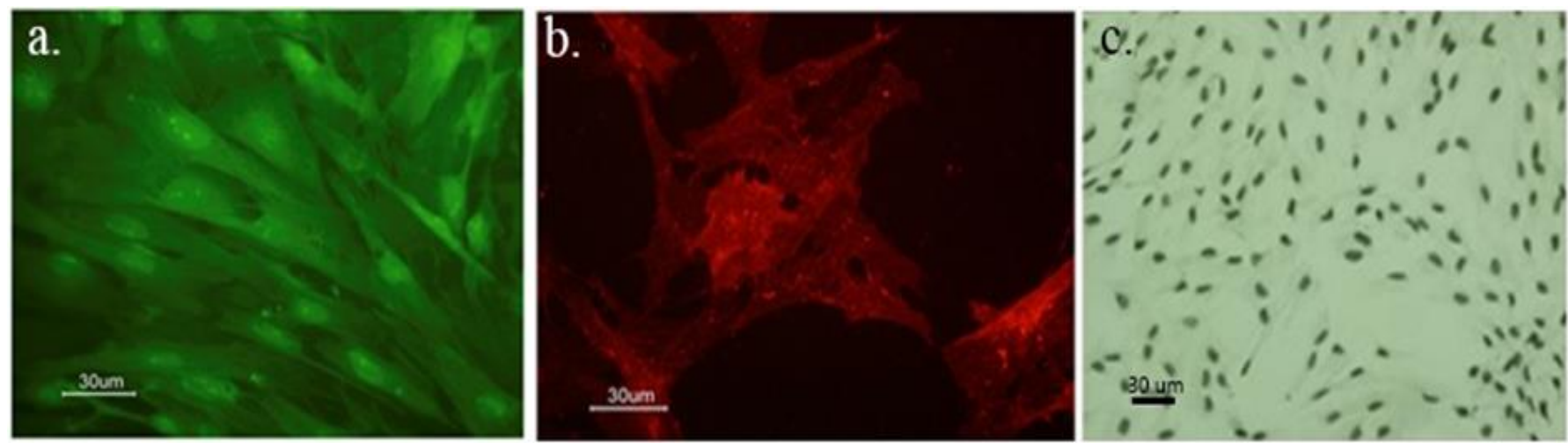

Figure 6. Cultured ADSCs expressed markers such as CD71 (a) and CD90 (b). More than $90 \%$ of the cultured cells were BrdU positive (c).
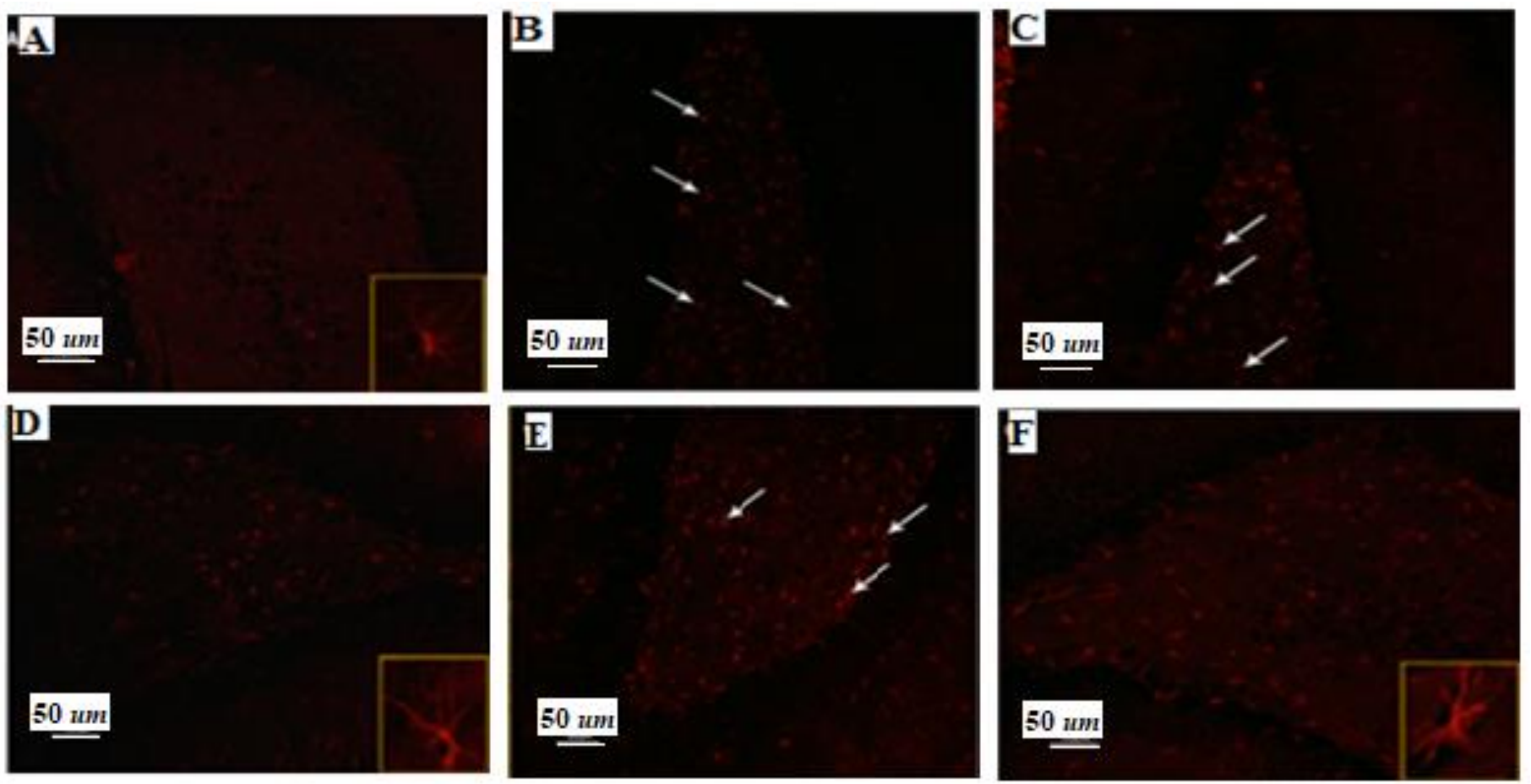

Figure 7. Immunohistochemical staining of GFAP-positive neurons in the hippocampus of Sham (A), Lesion (B), $\alpha$ MEM (C), Cell (D), Water (E) and Extract $(F)$ groups.
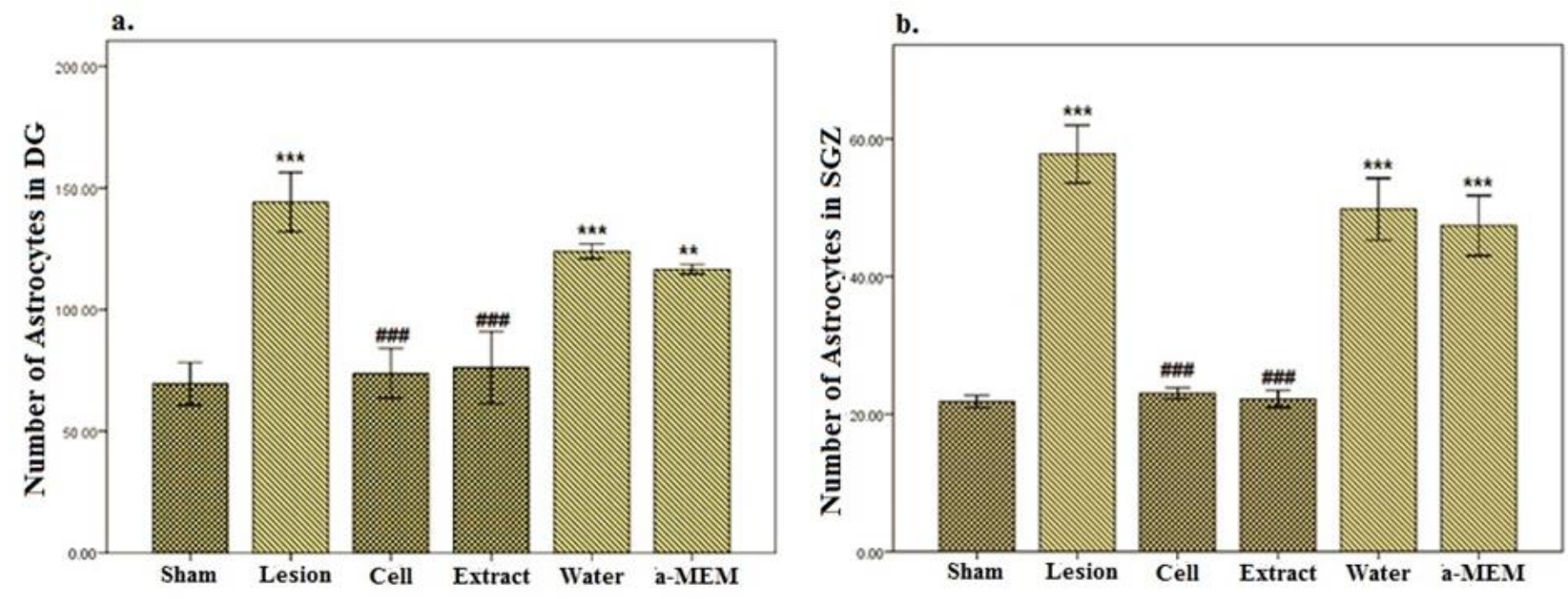

Figure 8. The number of GFAP positive astrocytes in DG (a) and SGZ (b) regions of the hippocampus. It was shown that the density of astrocytes in lesion, water and a-MEM were increased significantly compared to sham, cell and extract groups.

${ }^{* * *} p<0.000,{ }^{* *} p<0.00$ compared with sham. \#\#\# $p<0.000$ compared with lesion. Data are presented as mean $\pm S E M$.

Brazilian Archives of Biology and Technology. Vol.64: e21180392, 2021 www.scielo.br/babt 


\section{DISCUSSION}

In this study, spatial memory of Parkinsonian rats was evaluated using MWM. The results of the training phase, before lesion showed the ability of all animals to learn, as revealed by decrease in escape latency and distance traveled, from first to fourth day of training. All lesioned groups, except extract group, had lower learning ability than sham in training sessions, one week post lesion. By the other word, pretreatment with $\mathrm{RE}$ before lesion compensates the negative effects of injury on learning ability. The learning ability of cell treated rats was similar to sham group, it means that cell injection as pre-treatment with extract could improve spatial memory impairment in injured rats in the $3^{\text {rd }}$ and $4^{\text {th }}$ training sessions.

The process of memory retention was evaluated one day after the end of $3^{\text {rd }}$ and $4^{\text {th }}$ training sessions, which was performed 4 and 8 weeks after the lesion. There was no significant difference in the distance traveled between the groups, suggesting that the development of this model leads to memory impairment before motor disorder. Pre-treated animals with RE and also cell injected animals showed significant increase of time spent in target quadrant as compared with sham, in former probe tests. The results of this study clearly demonstrated the effects of cognitive improvement of RE and ADSCs in the Parkinson's model.

Rasoolijazi and coauthors reported the protective effect of carnosic acid against memory deficit in Alzheimer' disease [21]. Memory impairment of Parkinsonian rats was improved by oral administration of RE [22]. Schwerk in 2015, showed that ADSCs transplantation lead to improve cognitive performance [23].

RE has antioxidant, anti-tumor and anti-inflammatory activities. Carnosic acid, carnosol, rosemarinic acid and ursolic acid were the main polyphenolic compounds of extract. Among these compounds, carnosic acid exhibited a promoting antioxidant activity [24]. Reports also confirmed the anti-depressant, antiallergic, anti-inflammatory, anti-tumor, and anti-apoptotic effects of this extract [25]. Lee reported the antioxidant effect of extract on dopaminergic neurons and believed that rosemarinic acid with an antioxidant effect plays a neuroprotective role on dopaminergic neurons [25]. Also, Du and colleagues in 2010 showed the neuroprotective effects of RE against 6-OHDA and MPTP neurotoxins by anti-apoptotic and antioxidant properties in vitro [26]. Many researchers have focused on the effects of the RE and its components on aging or trauma induced cognitive dysfunctions, and showed its therapeutic potentials [2729].

Today, research on stem cells and their therapeutic use have been progressed. Mesenchymal stem cells can differentiate into many cell types including neural cells [30]. It was reported that labeled stromal cells injected into the striatum can express neuronal and glial markers and relatively improve PD symptoms. Yan et al. in 2013 showed that MSCs can inhibit inflammatory factors produced by active microglia and suppress microglial activity in vitro [31]. MSCs can also modulate the production of neurotrophic factors and subsequently, ameliorate neurological disorders [32].

In this study, we also evaluated the histological effects of RE and ADSCs on the hippocampus. There were studied in DG, CA1 and CA3 regions of the hippocampus. Cell counting showed that administration of $\mathrm{RE}$ at a dose of $50 \mathrm{mg} / \mathrm{kg}$, and also ADSCs transplantation at 8 weeks after 6-OHDA-induced models, significantly increased the number of granular cells in the DG region and also, the number of pyramidal cells in CA1 and CA3 regions compared with lesion, water and a-MEM groups, while the treated groups showed no significant difference of cell density as compared to sham. The results showed that the reduction of cell density induced by the 6-OHDA lesion was completely compensated by the treatment with the extract or stem cell transplantation. Thus, ADSCs were considered to have a therapeutic effect in PD.

It was also observed that the number of GFAP positive astrocytes increased in the lesion, $\alpha$-MEM and water groups compared with the sham group. Cell transplantation or RE reduced the density of GFAP positive cells as compared to the sham group. Therefore, the treatments not only improved the density of the granular and pyramidal cells of the damaged areas of the hippocampus, but also decreased the density of GFAP-positive astrocytes. Astrogliosis is an important response of astrocytes to infection, trauma, $\alpha$ synuclein accumulation, ischemia, and neurodegenerative diseases [33]. Astrogliosis and microgliosis in the SNc of patients with PD are the main features of the disease [34]. Activated glial cells can play a role in the death of dopaminergic neurons by activating apoptosis through cytokines (such as TNF- $\alpha$, IL- $\beta$, IL- 6 and interferon gamma, and production of nitric oxide), released into the neurons and stimulate lipid peroxidation, DNA breaks and inhibition of mitochondrial metabolism [35-37]. It was suggested that inhibition of astrocytes activity during inflammatory and damaging processes can be a therapeutic way to reduce neuronal damage during PD [38]. The immunohistochemical study of anti-GFAP showed that the number of GFAP positive astrocytes decreased in the animals treated with RE or ADSCs. 


\section{CONCLUSIONS}

The model used in this study was a suitable model for PD memory impairment. This model results in spatial learning and memory impairment through bilateral injection of 6-OHDA into the SNc which also leads to the destruction of neurons in the hippocampus. The Parkinsonian rats, treated with RE or ADSCs, appeared almost the same as the animals in the sham group in the behavioral, histological and immunohistochemical studies, and no significant difference was observed between the cells and REtreated groups. The results indicated that ADSCs transplantation or RE pre-treatment had neuroprotective effect and supported the recovery of memory deficit in 6-OHDA-induced rat model of PD.

Acknowledgments: This study was financially supported by Biological school of Damghan university. There is no conflict of interest in this article.

Conflicts of interest: The authors declare no conflict of interest.

\section{REFERENCES}

1. Ferro MM, Bellissimo MI, Anselmo-Franci JA, Angellucci ME, Canteras NS, Da Cunha C. Comparison of bilaterally 6-OHDA- and MPTP-lesioned rats as models of the early phase of Parkinson's disease: histological, neurochemical, motor and memory alterations. J Neurosci. Methods. 2005;148(1):78-87.

2. Hely MA, Morris JG, Reid WG, Trafficante R. Sydney Multicenter Study of Parkinson's disease: non-L-doparesponsive problems dominate at 15 years. Mov. Disord. 2005;20(2):190-9.

3. Vajda FJ. Neuroprotection and neurodegenerative disease. J. Clin.Neurosci. 2002;9(1):4-8.

4. Martig AK, Mizumori SJ. Ventral tegmental area and substantia nigra neural correlates of spatial learning. Learn. Mem. 2011;18(4):260-71.

5. Kim SJ, Kim JS, Cho HS, Lee HJ, Kim SY, Kim S, et al. Carnosol, a component of rosemary (Rosmarinus officinalis L.) protects nigral dopaminergic neuronal cells. Neuroreport. 2006;17(16):1729-33.

6. Wu CR, Tsai CW, Chang SW, Lin CY, Huang LC, Tsai CW. Carnosic acid protects against 6-hydroxydopamineinduced neurotoxicity in in vivo and in vitro model of Parkinson's disease: involvement of antioxidative enzymes induction. Chem. Biol. Interac. 2015;225:40-6.

7. Wang J, Xu H, Jiang H, Du X, Sun P, Xie J. Neurorescue effect of rosmarinic acid on 6-hydroxydopaminelesioned nigral dopamine neurons in rat model of Parkinson's disease. J. Mol. Neurosci. 2012;47(1):113-9.

8. Ito N, Yabe T, Gamo Y, Nagai T, Oikawa T, Yamada H, et al. Rosmarinic acid from Perillae Herba produces an antidepressant-like effect in mice through cell proliferation in the hippocampus. Bio. Pharm. Bull. 2008;31(7):1376-80.

9. Lin CY, Tsai CW. Carnosic Acid Attenuates 6-Hydroxydopamine-Induced Neurotoxicity in SH-SY5Y Cells by Inducing Autophagy Through an Enhanced Interaction of Parkin and Beclin1. Mol. Neurobiol. 2017;54(4):281322.

10. Ma Y, Peng S, Dhawan V, Eidelberg D. Dopamine cell transplantation in Parkinson's disease: challenge and perspective. Br. Med. Bull. 2011;100:173-89.

11. Wijeyekoon R, Barker RA. Cell replacement therapy for Parkinson's disease. Biochim Biophys Acta. 2009;1792(7):688-702.

12. Mizuno $\mathrm{H}$. Adipose-derived stem cells for tissue repair and regeneration: ten years of research and a literature review. J. Nippon. Med. Sch. 2009;76(2):56-66.

13. Abdanipour A, Tiraihi T, Delshad A. Trans-differentiation of the adipose tissue-derived stem cells into neuron-like cells expressing neurotrophins by selegiline. Iran. Biomed. J. 2011;15(4):113-21.

14. Mizuno H, Tobita M, Uysal AC. Concise review: Adipose-derived stem cells as a novel tool for future regenerative medicine. Stem Cells. 2012;30(5):804-10.

15. Chen Y, Ai Y, Slevin JR, Maley BE, Gash DM. Progenitor proliferation in the adult hippocampus and substantia nigra induced by glial cell line-derived neurotrophic factor. Exp. Neurol. 2005;196(1):87-95.

16. Hoglinger GU, Arias-Carrion $\mathrm{O}$, Ipach B, Oertel WH. Origin of the dopaminergic innervation of adult neurogenic areas. J. Comp. Neurol. 2014;522(10):2336-48.

17. Nagano-Saito A, Habak C, Mejia-Constain B, Degroot C, Monetta L, Jubault T, et al. Effect of mild cognitive impairment on the patterns of neural activity in early Parkinson's disease. Neurobiol. Aging. 2013.

18. Bibb JA. Decoding dopamine signaling. Cell. 2005;122(2):153-5.

19. Wattanathorn J, Sutalangka C. Laser Acupuncture at HT7 Acupoint Improves Cognitive Deficit, Neuronal Loss, Oxidative Stress, and Functions of Cholinergic and Dopaminergic Systems in Animal Model of Parkinson's Disease. eCAM. 2014;2014:937601. 
20. Friedman T. The Effect of Rosmarinic Acid on Immunological and Neurological Systems. J. Restor. Med. 2015;4(1):50-9.

21. Rasoolijazi H, Azad N, Joghataei MT, Kerdari M, Nikbakht F, Soleimani M. The protective role of carnosic acid against beta-amyloid toxicity in rats. Sci. World. J. 2013;2013:917082.

22. Rasoul A, Maryam HG, Taghi GM, Taghi L, dehghan Ra. Antioxidant Activity of Oral Administration of Rosmarinus Officinalis Leaves Extract on Rat's Hippocampus which Exposed to 6-Hydroxydopamine. Braz. Arch. Biol. Technol. 2016;59:e16150354.

23. Schwerk A, Altschuler J, Roch M, Gossen M, Winter C, Berg J, et al. Adipose-derived human mesenchymal stem cells induce long-term neurogenic and anti-inflammatory effects and improve cognitive but not motor performance in a rat model of Parkinson's disease. Regen. Med. 2015;10(4):431-46.

24. De Oliveira MR. Carnosic Acid as a Promising Agent in Protecting Mitochondria of Brain Cells. Mol. Neurobiol. 2018;55:6687-99.

25. Lee HJ, Cho HS, Park E, Kim S, Lee SY, Kim CS, et al. Rosmarinic acid protects human dopaminergic neuronal cells against hydrogen peroxide-induced apoptosis. Toxicology. 2008;250(2-3):109-15.

26. Du T, Li L, Song N, Xie J, Jiang H. Rosmarinic acid antagonized 1-methyl-4-phenylpyridinium (MPP+)-induced neurotoxicity in MES23.5 dopaminergic cells. Int. J. Toxicol. 2010;29(6):625-33.

27. Farr SA, Niehoff ML, Ceddia MA, Herrlinger KA, Lewis BJ, Feng S, et al. Effect of botanical extracts containing carnosic acid or rosmarinic acid on learning and memory in SAMP8 mice. Physiol. Behav. 2016;165:328-38.

28. Rasoolijazi H, Mehdizadeh M, Soleimani M, Nikbakhte F, Eslami Farsani M, Ababzadeh S. The effect of rosemary extract on spatial memory, learning and antioxidant enzymes activities in the hippocampus of middleaged rats. Med. J. Islam Repub Iran. 2015;29:187.

29. Song $\mathrm{H}, \mathrm{Xu} \mathrm{L}$, Zhang $\mathrm{R}$, Cao Z, Zhang H, Yang L, et al. Rosemary extract improves cognitive deficits in a rats model of repetitive mild traumatic brain injury associated with reduction of astrocytosis and neuronal degeneration in hippocampus. Neurosci. Lett. 2016;622:95-101.

30. Pansky A, Roitzheim B, Tobiasch E. Differentiation potential of adult human mesenchymal stem cells. Clin. Lab. 2007;53(1-2):81-4.

31. Yan K, Zhang R, Sun C, Chen L, Li P, Liu Y, et al. Bone marrow-derived mesenchymal stem cells maintain the resting phenotype of microglia and inhibit microglial activation. PloS one. 2013;8(12):e84116.

32. Al Jumah MA, Abumaree MH. The Immunomodulatory and Neuroprotective Effects of Mesenchymal Stem Cells (MSCs) in Experimental Autoimmune Encephalomyelitis (EAE): A Model of Multiple Sclerosis (MS). Int. J. Mol. Sci. 2012;13(7):9298-331.

33. Liddelow SA, Barres BA. Reactive Astrocytes: Production, Function, and Therapeutic Potential. Immunity. 2017;46(6):957-67.

34. Fricke IB, Viel T, Worlitzer MM, Collmann FM, Vrachimis A, Faust A, et al. 6-hydroxydopamine-induced Parkinson's disease-like degeneration generates acute microgliosis and astrogliosis in the nigrostriatal system but no bioluminescence imaging-detectable alteration in adult neurogenesis. Eur. J. Neurosci. 2016;43(10):135265.

35. Booth HDE, Hirst WD, Wade-Martins R. The Role of Astrocyte Dysfunction in Parkinson's Disease Pathogenesis. Trends. Neurosci. 2017;40(6):358-70.

36. Episcopo FL, Tirolo C, Testa N, Caniglia S, Morale MC, Marchetti B. Reactive astrocytes are key players in nigrostriatal dopaminergic neurorepair in the MPTP mouse model of Parkinson's disease: focus on endogenous neurorestoration. Curr. Aging Sci. 2013;6(1):45-55.

37. Machado A, Herrera AJ, Venero JL, Santiago M, de Pablos RM, Villaran RF, et al. Inflammatory Animal Model for Parkinson's Disease: The Intranigral Injection of LPS Induced the Inflammatory Process along with the Selective Degeneration of Nigrostriatal Dopaminergic Neurons. ISRN. Neurol. 2011;2011:476158.

38. Rappold PM, Tieu K. Astrocytes and therapeutics for Parkinson's disease. Neurotherapeutics. 2010;7(4):413-23.

(C) 2021 by the authors. Submitted for possible open access publication under the terms and conditions of the Creative Commons Attribution (CC BY NC) license (https://creativecommons.org/licenses/by-nc/4.0/). 